\title{
DNA methylation of neural EGFL like 1 (NELL1) is associated with advanced disease and the metastatic state of renal cell cancer patients
}

\author{
INGA PETERS $^{1}$, NATALIA DUBROWINSKAJA ${ }^{1}$, JÖRG HENNENLOTTER ${ }^{2}$, WIEBKE INGA ANTONOPOULOS ${ }^{3}$, \\ CHRISTOPH-A.J. VON KLOT ${ }^{1}$, HOSSEIN TEZVAL ${ }^{1}$, ARNULF STENZL ${ }^{2}$, \\ MARKUS A. KUCZYK ${ }^{1}$ and JÜRGEN SERTH ${ }^{1}$
}

\begin{abstract}
${ }^{1}$ Department of Urology, Hannover Medical School, D-30625 Hannover; ${ }^{2}$ Department of Urology, Eberhard-Karls University, D-72074 Tübingen; ${ }^{3}$ Department of Pathology, Hannover Medical School, D-30625 Hannover, Germany
\end{abstract}

Received January 29, 2018; Accepted August 17, 2018

DOI: 10.3892/or.2018.6732

\begin{abstract}
Recent studies have shown that NELL1 expression is silenced epigenetically in human renal cell cancer (RCC) tissues and in RCC cell lines. However, it remains unknown whether NELL1 promoter methylation observed in clinical specimens might be associated with the clinicopathology or survival of patients with RCC. We analyzed NELL1 DNA methylation in tissues from patients with RCC and in adjacent normal renal tissues. In addition, we evaluated NELL1 methylation in cell lines derived from different urogenital tumors (prostate cancer, urothelial cancer and RCC). We performed regression analyses to determine whether NELL1 methylation is associated with clinicopathological parameters and recurrence-free survival (RFS). This cross-sectional study included 98 patients with RCC and 63 paired tumor and adjacent normal tissue samples. We analyzed a locus in the intron 1 region of $N E L L 1$ with pyrosequencing. We performed in silico analysis of NELL1 methylation in the TCGA Kidney Renal Clear Cell Carcinoma (KIRC) data set ( $\mathrm{n}=284$ patients), which served as a validation study. Statistical analyses were performed with the two-sided paired t-test for paired tumor and adjacent normal samples. We used logistic regression for subgroup comparisons and Cox regression for RFS comparisons. The mean methylation level was $6.8 \%$ higher in RCC tissues compared to paired adjacent normal tissues (paired t-test, $\mathrm{P}<0.001$ ). Methylation levels in RCC were associated with advanced disease $(\mathrm{P}=0.002)$, the presence of distant metastases $(\mathrm{P}=0.004)$, and shorter RFS ( $\mathrm{P}=0.035$, HR: 4.15). In silico validation with TCGA KIRC data for adjacent loci also demonstrated that
\end{abstract}

Correspondence to: Dr Jürgen Serth, Department of Urology, Hannover Medical School, OE6247, Carl-Neuberg-Str. 1, D-30625 Hannover, Germany

E-mail: serth.juergen@mh-hannover.de

Key words: NELL1, advanced renal cell carcinoma, hypermethylation, epigenetic silencing, metastasis and survival high relative methylation levels were associated with adverse clinicopathology and shortened RFS. Our results suggest that NELL1 methylation contributes to RCC disease progression. This finding could provide a clinical marker to complement recent functional analyses in tumor models.

\section{Introduction}

Renal cell cancer (RCC) is the twelfth most common cancer worldwide; in 2012, it accounted for about 338,000 new cases (1). A comprehensive characterization of the molecular alterations associated with RCC was recently carried out by the TCGA Kidney consortium (KIRC) (2). Notably, they described only limited associations between gene mutations and clinical or survival parameters in RCC patients. Thus, it was questionable whether the translation of genetic mutations or variant information would be useful for future personalized diagnoses, prognoses, or therapy predictions. This question was consistent with earlier genetic studies, which demonstrated inconsistent results concerning associations with clinical pathology or patient survival (3). In contrast, the KIRC data, like many previous studies, reported that clear cell (cc) RCC typically showed features of epigenetic alterations, such as hypermethylation of gene promoters and concurrent loss of gene function, due to transcriptional silencing. Indeed, it has been consistently observed, in a substantial number of genes, that DNA hypermethylation occurs with high frequency, and the degree of hypermethylation is correlated with clinical and pathological features of patients. In addition, the tumor suppressors known as RAS-associated domain family 1 (RASSF1A) and secreted frizzled-related protein 1 (SFRP1) were each found in $30 \%$ to $>70 \%$ of tumors. These tumor suppressors were associated with both clinical pathological features and outcomes (4-7). In RCC, a significant incidence of epigenetic alterations has been found in the COL1A1, IGFBPI, EDNRB and KRT19 genes, in cell lines and primary tumors $(8,9)$. A recent report demonstrated tumor-specific hypermethylation in RCC, which was found to be correlated with adverse pathology and was associated with overall survival among patients undergoing targeted therapy (10). 
In view of the fact that a multitude of hypermethylated genes (11) have been described and that the KIRC data provide a large number of new candidate methylated loci for ccRCC, the question arises, how can we identify the most relevant genes? Based on traditional genetic approaches, we might assume that the most promising candidates for future translational purposes would be genes that are associated with functional losses, high alteration frequencies in tumors, and altered clinical behaviors in patients. Here, we focused on the clinical relevance of DNA methylation in the neural epidermal growth factor-like 1 (NELL1) gene. NELL1 is a multimeric extracellular glycoprotein, originally identified in neural tissues (12). The gene is located on chromosome 11p15.1, and it is involved in osteogenic differentiation in mammals and bone regeneration in defects of the calvarium $(13,14)$.

NELL1 expression has been detected in normal embryonic kidney, brain and prostate, and in bladder cancer, lymphomas and different cancer cell lines (15-18). A recent study revealed that NELL1 protein expression was reduced in RCC tissues (19). Considering that promoter hypermethylation and diminished mRNA expression were detected in RCC cell lines, transcriptional silencing was assumed to explain low NELL1 expression in RCC (19).

No studies have described NELL1 promoter methylation in RCC tissues or its correlation to clinicopathological parameters or clinical outcome. Here, we investigated NELL1 promoter methylation in cancerous and adjacent non-cancerous tissues derived from patients with RCC and a subset of patients with ccRCC and in 18 malignant and benign renal cancer cell lines. Results were confirmed in silico with the KIRC data set.

\section{Materials and methods}

Tissue specimens. Ninety-eight tumor tissue samples were obtained from kidney surgeries carried out between 2001 and 2005 at Eberhard Karls University, Tübingen. Tissue sampling, storage, and processing were performed as described previously (20). Patient characteristics are provided in Table I. Pairs of tissue samples from tumors (TU) and adjacent tumor-free tissue $(\operatorname{adN})$ were obtained from 63 patients.

The Ethics Committee of the institution of Eberhard Karls University approved the study (vote no. 128/2003V and 1213-2011) and informed consent was obtained from each patient. Two pathologists evaluated all specimens, with respect to tumor stage, grade and histological subtypes. Tumor stages were assessed according to the UICC 2002 issue of the TNM system, and nuclear grading was based on the Fuhrman grading system (21). Histological subtypes were assessed according to the consensus classification of RCC. Localized RCC was defined as $\mathrm{pT} \leq 2$, N0/M0; advanced tumors were defined as $\mathrm{pT} \geq 3$ and/or N1/M1. Low-stage tumors were defined as the group of pT1 and pT 2 tumors; high stage tumors were defined as the group of pT3 and T4 tumors. Follow-up data were available for 24 patients. The duration of the follow-up was calculated from the date of surgery to the date of recurrence, progression, death, or the last follow-up.

Primary and cancer cell lines. Prostate cancer (LN-cap, DU-145), urothelial cancer (CLS439, HB-CLS2, HB-CLS1,
Table I. Clinicopathological parameters of the discovery and evaluation cohort.

\begin{tabular}{|c|c|c|}
\hline & Hannover cohort & TCGA cohort \\
\hline Total cases & 98 & 284 \\
\hline \multicolumn{3}{|l|}{ Sex } \\
\hline Female & 35 & 96 \\
\hline Male & 63 & 188 \\
\hline \multicolumn{3}{|l|}{ Age (years) } \\
\hline Median & 64 & 61 \\
\hline Minimum & 35 & 26 \\
\hline Maximum & 91 & 90 \\
\hline \multicolumn{3}{|l|}{ T-classification } \\
\hline pT1 & 10 & 15 \\
\hline pT1a & 26 & 72 \\
\hline pT1b & 17 & 46 \\
\hline pT2 & 7 & 32 \\
\hline pT3 & 3 & 3 \\
\hline pT3a & 9 & 65 \\
\hline pT3b & 22 & 38 \\
\hline $\mathrm{pT} 3 \mathrm{c}$ & 1 & NA \\
\hline pT4 & 1 & 8 \\
\hline \multicolumn{3}{|l|}{ Lymph node status } \\
\hline No & 85 & 127 \\
\hline N1 & 13 & 9 \\
\hline $\mathrm{Nx}$ & NA & 148 \\
\hline \multicolumn{3}{|l|}{ Distant metastasis } \\
\hline M0 & 79 & 232 \\
\hline M1 & 19 & 52 \\
\hline \multicolumn{3}{|l|}{ Differentiation } \\
\hline G1 & 22 & 5 \\
\hline G1-2 & 14 & NA \\
\hline $\mathrm{G} 2$ & 48 & 117 \\
\hline G2-3 & 6 & NA \\
\hline G3 & 8 & 112 \\
\hline G4 & NA & 48 \\
\hline Gx & NA & 2 \\
\hline \multicolumn{3}{|l|}{ Status of disease } \\
\hline Localized $^{\mathrm{a}}$ & 51 & NA \\
\hline Advanced $^{b}$ & 46 & NA \\
\hline Paired tissue samples ${ }^{c}$ & 63 & 160 \\
\hline
\end{tabular}

${ }^{\mathrm{a}} \mathrm{pT} \leq 2, \mathrm{~N} 0, \mathrm{M} 0 .{ }^{\mathrm{b}} \mathrm{pT} \geq 3$ and/or N1, M1. ${ }^{\mathrm{c}}$ Tumor and adjacent normal renal tissue. NA, not available.

5637), and RCC (ACHN, A498, 786-O, RCC-GS, RCC-HS, RCC-MF) cell lines were purchased from Lonza (Basel, Switzerland). Primary normal cell lines (RPTEC, primary renal proximal tubular epithelial cells) were acquired from Cell Lines Services (CLS, Eppelheim, Germany). All cells were cultured according to the manufacturer's protocol and as described previously $(20,22)$. 


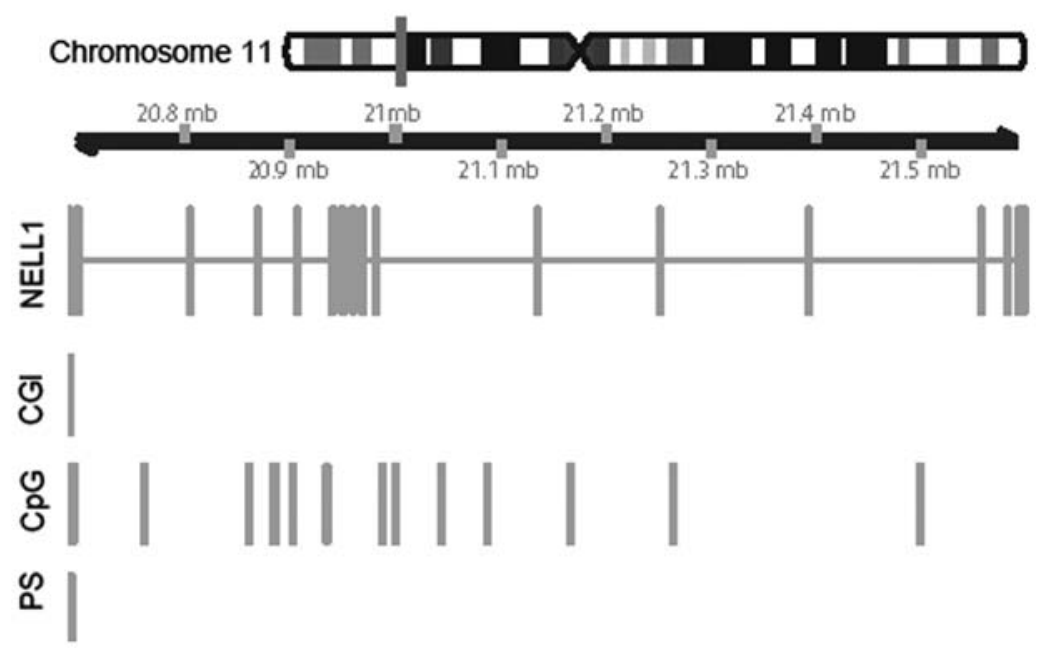

Figure 1. NELL1 gene information. Schematic Figure of chromosome 11 with information concerning the position of the NELL1 gene and the NELL1 transcript used for pyrosequencing (PS), in relation to the $\mathrm{CpG}$-island (CGI) and $\mathrm{CpG}$ sites (CpG).

DNA extraction and bisulfite conversion. DNA extraction, bisulfite conversion, and control of tumor cell content were performed as reported previously (22). Total DNA was extracted from 20 serial frozen sections $(20 \mu \mathrm{m})$ from each tumoral and normal specimen after proteinase $\mathrm{K}$ digestion, with the standard phenol/chloroform extraction method. Each $1 \mu \mathrm{g}$ of extracted DNA was then subject to bisulfite conversion, as described previously, and aliquots were stored at $-20^{\circ} \mathrm{C}$ (5). Additionally, two serial sections of each tissue sample were stained with hematoxylin-eosin and re-evaluated by pathologists.

Bisulfite-pyrosequencing for NELL1 promoter methylation analysis. We performed methylation analyses of ten NELL1 CpG sites (base positions: 20,587, 591, 596, 604, 611, $\sim 615, \sim 618, \sim 626, \sim 634, \sim 636, \sim 691)$ within the $\mathrm{CpG}$ island (CGI), which extended over the 5'UTR and exon 1 of the NELL1 gene (Fig. 1, 'CGI' and 'Pyro'). Analyses were performed with bisulfite-pyrosequencing, according to the manufacturer's protocol (Qiagen, Hilden, Germany) and as described previously $(6,23)$. We used a two-step PCR protocol. For the first step, the primers were: Forward (5'-GGGATGAATTGTGGT AATT-3') and reverse (5'-GGGACACCGCTGATCGTTTAC TACRAAAATCTAAACTACAAA-3'). For the second step, the primers were: Forward (5'-GGAAGTAAAGAGGGTAAT ATTG-3') and reverse (Biotin-5'-GGGACACCGCTGATC GTTTA-3'). Measurements were performed with the PyroMark Q24 pyrosequencing system (Qiagen, Hilden, Germany).

Statistical analysis. Statistical analyses of NELL1 methylation levels in paired TU and adN tissue samples were performed with the two-sided paired t-test. Tumor subgroups were assessed with univariate logistic regression analysis and dichotomization, as specified. Visual comparisons of subgroups were performed with notched boxplots, which show the median, the $25 \%$ quartiles, and the estimated confidence intervals (notches). Univariate Cox regression analyses were performed to examine recurrence-free survival (RFS). Hazard ratios (HR), $95 \%$ confidence intervals $(95 \% \mathrm{CI}$ ), and P-values were calculated. Survival was evaluated with the Kaplan-Meier analysis. All analyses were performed with $\mathrm{R}$ version 3.2 . The 'maxRank' package was used to calculate the optimum cutoff for the survival analysis (24). Therefore, the corresponding value of $15 \%$ is a result of a mathematical calculation in $\mathrm{R}$ using the Package 'OptimalCutpoint'.

P-values $<0.05$ were considered to indicate statistical significance. In silico validation was carried out with the subset of level 3 data from the KIRC data set, annotated for NELL1. Distributions of the methylation differences obtained for each pair of TU and corresponding adN samples were calculated for each annotated $\mathrm{CpG}$ site. We used quality criteria for statistical information on the sites. Consequently, only $\mathrm{CpG}$ sites that showed approximately normal or bimodal density distributions were considered for further evaluation. We excluded $\mathrm{CpG}$ sites characterized by methylation differences that exhibited more or less discrete and constant values, in the paired tissue group comparisons.

\section{Results}

NELL1 methylation in tumor cell line models. Twelve cell lines that represented urological cancer models and one normal tissue cell model (RPTECs) were analyzed for NELL1 methylation. RPTECs demonstrated low relative methylation values $(\sim 3 \%)$ and a large number of kidney tumor cell lines, including A498, RCC-HS, and RCC-GS, displayed high relative methylation (30-60\%; Fig. 2). Two kidney cancer cell lines (786-O and $\mathrm{ACHN}$ ) displayed medium relative methylation (10-20\% maximum). The prostate cancer cell lines, LN-cap and DU-145, exhibited elevated methylation, and two (HB-CLS2, CLS-439) out of four urothelial cancer cell lines displayed high methylation ( 40\%, Fig. 2).

NELL1 DNA hypermethylation in paired tumor and adjacent normal renal tissue samples. To investigate whether NELL1 showed tumor-specific DNA hypermethylation, we compared TU paired with adN tissue samples. We found that TU tissues displayed a significantly higher mean methylation level $(6.8 \%)$ compared to paired adN tissues (paired t-test, $\mathrm{P}=1.64 \mathrm{e}^{-5}$, Fig. 3). 


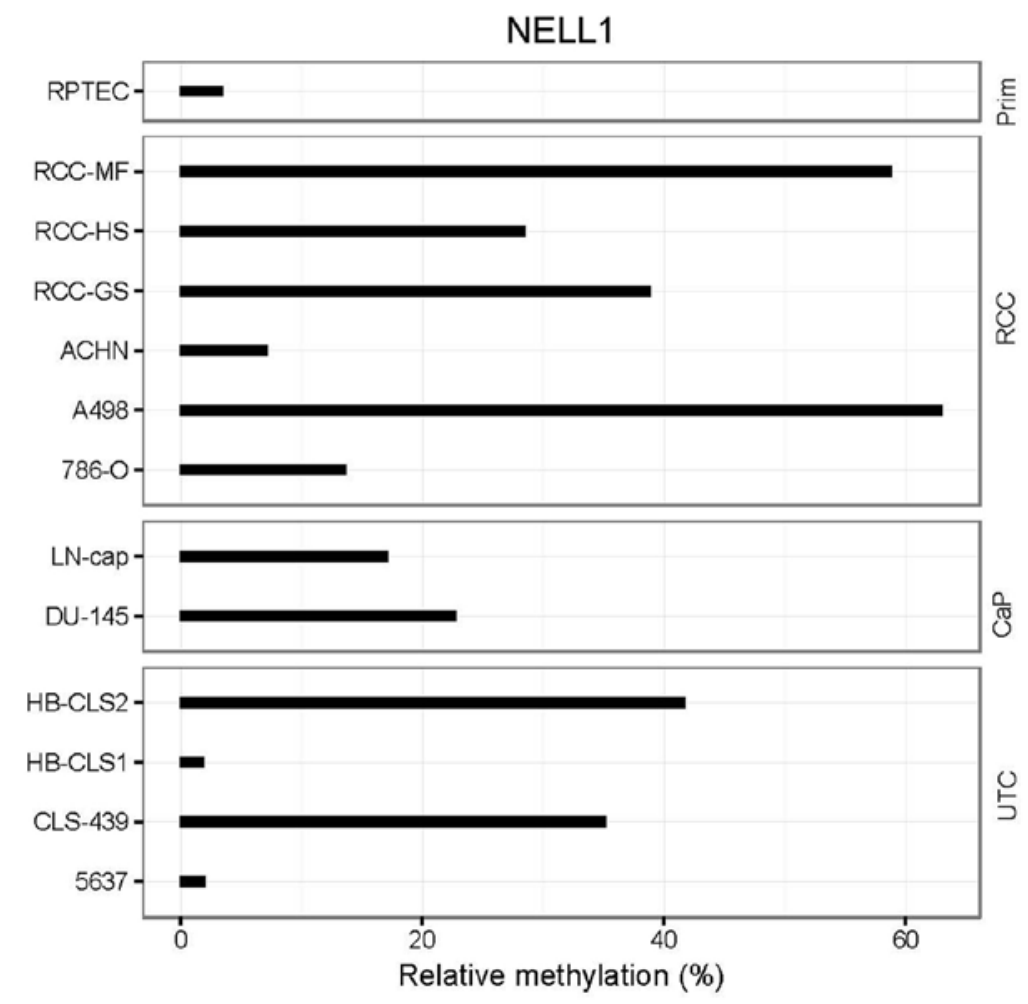

Figure 2. Methylation of NELL1 in urological cancer cell lines. The diagram shows the relative methylation of $N E L L 1$ measured in six renal cell cancer (RCC), two prostate cancer (CaP), and four urothelial cancer (UTC) cell lines. A normal kidney cell line (RPTEC) was used as a negative control.

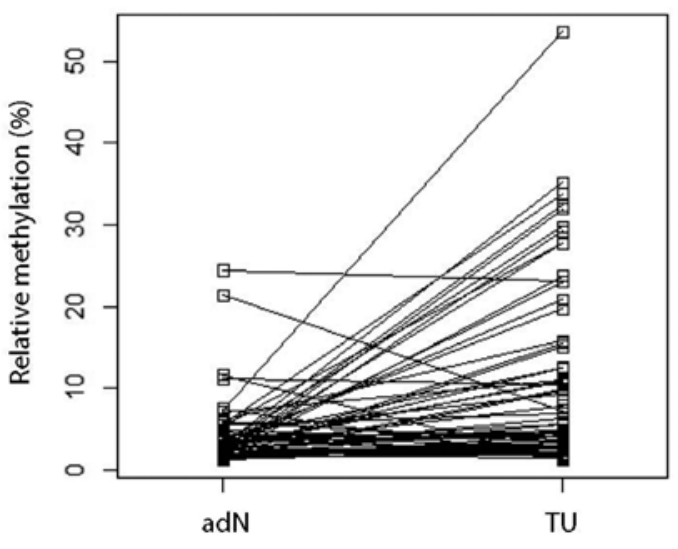

Figure 3. Methylation of NELL1 in paired tumor and normal tissue samples. Comparison of relative NELL1 methylation values in tumor (TU) and adjacent normal (adN) tissues acquired from patients with renal cell cancer (RCC). The mean relative methylation level is significantly higher in $\mathrm{TU}$ $(6.8 \%)$ compared to adN $(\mathrm{P}<0.001)$.

NELL1 DNA methylation and clinicopathological parameters. To investigate whether the clinicopathological parameters of the patients might be associated with altered NELL1 DNA methylation, patients were grouped according to different clinicopathological parameters, and then subjected to univariate logistic regression analyses, following the corresponding dichotomization. We found that methylation was significantly increased in the group with advanced tumors (mean methylation $12.6 \%$ ) compared to those with localized disease [6.1\%; $\mathrm{P}=0.002$; odds ratio $(\mathrm{OR})=1.08$; Fig. $4 \mathrm{~A}]$. Furthermore, the presence of distant metastases in patients was associated with a higher mean methylation (15.8\%) compared to patients without clinically detectable distant metastasis $(7.8 \%, \mathrm{P}=0.004$; OR: 1.07; Fig. 4B). Low- and high-stage tumor groups had mean methylation values of 7.5 and $11.8 \%$, respectively $(\mathrm{P}=0.044$; OR: 1.05). No significant difference in mean methylation was observed between groups that exhibited low and high histological tumor grades (Table II).

NELL1 promoter methylation and recurrence-free survival. Kaplan Meier and Cox regression analyses were performed to assess the relationship between NELL1 DNA methylation and the RFS of patients. We dichotomized patients according to the degree of DNA methylation, based on a cut-off value of $15 \%$ relative methylation. The Kaplan Meier analysis indicated that high NELL1 methylation was associated with a worse RFS (Fig. 5). The univariate Cox regression analysis identified NELL1 methylation as a significant risk factor for RFS (HR: 4.15, 95\% CI: 1.10-15.6; $\mathrm{P}=0.035)$.

In silico validation with KIRC data. We assessed the KIRC data set for associations between tumor-specific hypermethylation, clinicopathological parameters and survival, in an independent patient cohort. Out of 15 annotated CpG sites selected in a pre-analysis quality check (see Materials and methods), five showed hypomethylation (paired t-test, $\mathrm{P}<0.05$, adjusted with Bonferroni-Hochberg for multiple testing) and six showed hypermethylation $(\mathrm{P}<0.05)$. We observed significant associations between the methylation state and the state of metastasis in seven out of $15 \mathrm{CpG}$ sites (univariate logistic regression: $\mathrm{P}=1.8 \times 10^{-2}$ to $7.3 \times 10^{-7}$, OR: $\left.12-546\right)$. Four of these $\mathrm{CpG}$ sites were located within or adjacent to the CGIs 
Table II. Overview of NELL1 DNA methylation analyses in primary RCC tissue samples, and statistical comparison with clinicopathological parameters.

\begin{tabular}{lccccc}
\hline NELL1 methylation & \multicolumn{2}{c}{$\begin{array}{c}\text { Mean relative } \\
\text { methylation (\%) }\end{array}$} & P-value & Odds ratio & 95\% confidence interval \\
\hline Localized/advanced $^{\mathrm{a}}$ & 6.07 & 12.62 & $\mathbf{0 . 0 0 2}$ & 1.08 & $1.03-1.15$ \\
Low/high grade $^{\mathrm{b}}$ & 8.60 & 13.63 & 0.096 & 1.04 & $0.99-1.09$ \\
Distant metastasis (M0 vs. M1) $^{\text {Low/high stage }}$ & 7.75 & 15.85 & $\mathbf{0 . 0 0 4}$ & 1.07 & $1.02-1.12$ \\
& 7.48 & 11.76 & $\mathbf{0 . 0 4 4}$ & 1.05 & $1.00-1.09$ \\
\hline
\end{tabular}

${ }^{\mathrm{a} L o c a l i z e d ~ d i s e a s e: ~} \mathrm{pT} \leq 2, \mathrm{~N} 0, \mathrm{M} 0$; advanced disease: $\mathrm{pT} \geq 3$ and/or N1, M1, ${ }^{\mathrm{b} L o w}$ grade (G1/G1-2); high grade (G2-3/G3). ${ }^{\mathrm{c}} \mathrm{Low}$ stage (pT1/T2), high stage (pT3/T4). ${ }^{d}$ Univariate logistic regression. P-values denoted in bold print indicate statistical significance. RCC, renal cell cancer.
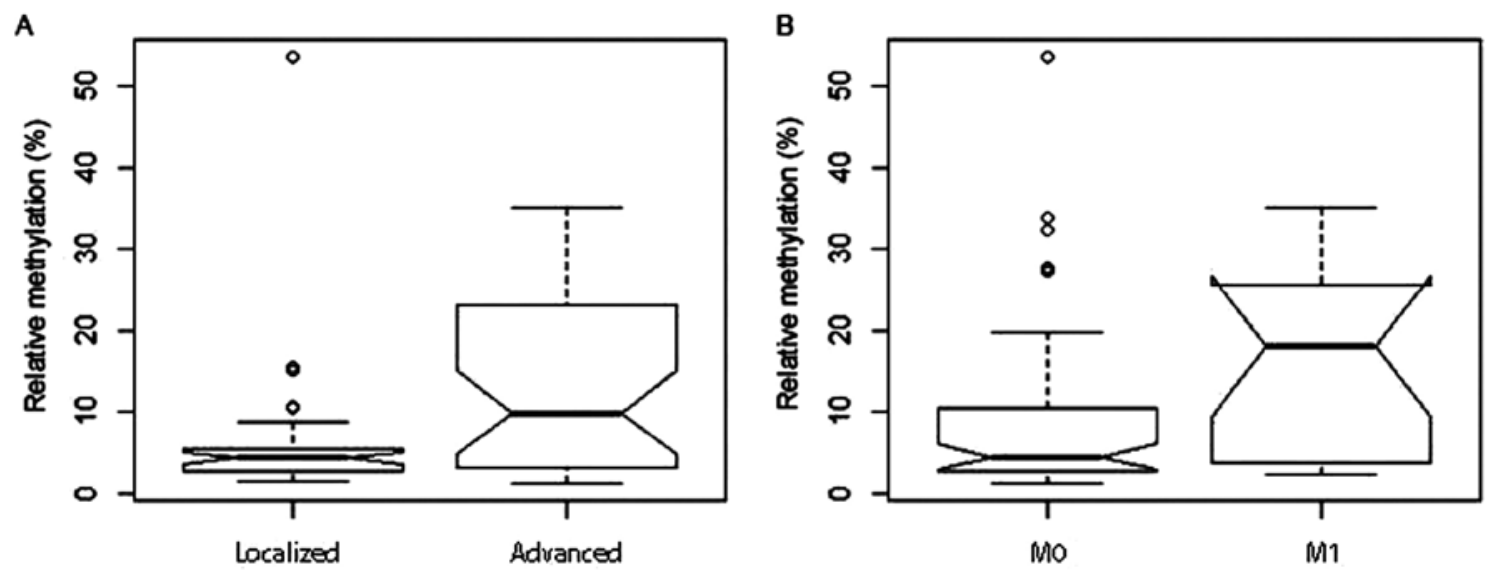

Figure 4. Box plot illustration of NELL1 methylation in renal cell cancer (RCC). Distribution of relative NELL1 DNA methylation levels among patients with RCC that exhibited (A) localized or advanced disease, and (B) the presence (M1) or absence (M0) of distant metastases. The notched box plot illustrates the median, the estimated confidence intervals, and the $25 \%$ quartiles in both groups. Statistical analyses demonstrated significantly higher mean methylation levels in advanced and M1 groups (see Table II).

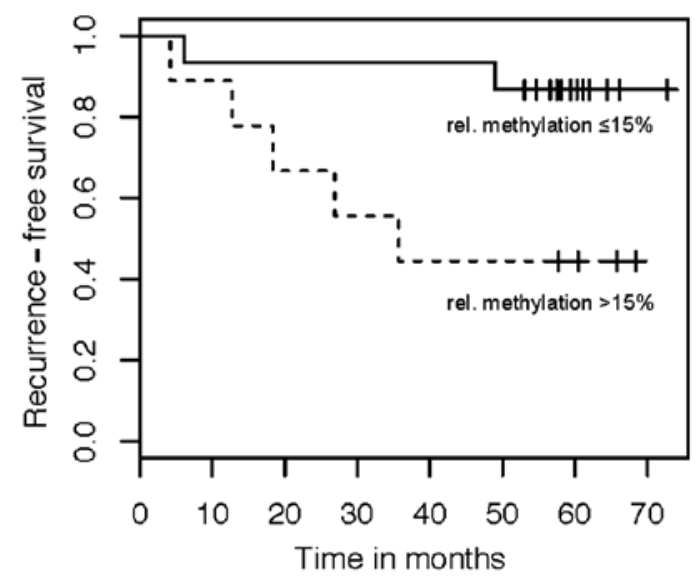

Figure 5. Kaplan-Meier survival analysis. Recurrence-free survival (RFS) in patients with methylation levels above the calculated cut-off point (dotted line) and in patients with methylation levels below the cut-off point (continuous line). The optimal calculated cut-off point was $15 \%$ methylation.

presented in Fig. 6. Notably, the corresponding CpG sites were also associated with high-stage tumors, high-grade tumors, and the RFS of patients (Fig. 6).

\section{Discussion}

NELL1 DNA methylation and epigenetic silencing of gene expression were previously described in RCC cell lines, which suggested that epigenetic alterations were potentially relevant to RCC pathology (19). In the present study, we aimed to ascertain whether NELLI DNA methylation was associated with clinicopathological parameters and disease outcome in patients with ccRCC. Comparisons between tumors (TU) and adjacent tumor-free tissue (adN) clearly demonstrated that tumor-specific hypermethylation occurred in the loci analyzed. An interrogation of the KIRC data set showed that several CpG sites in the extended CGI region at exon 1 of the gene also exhibited clear hypermethylation, which confirmed results from former cell line experiments and from the present study. Of note, the DNA methylation observed in cell lines probably reflected both intra-tumoral and inter-patient variability at each locus investigated. Indeed, both baseline methylation and age-dependent methylation show considerable variation among different organ systems. Thus, some of the observed differences in methylation reflected the normal biological variations in methylation expected among different tissues and tissue donors. 


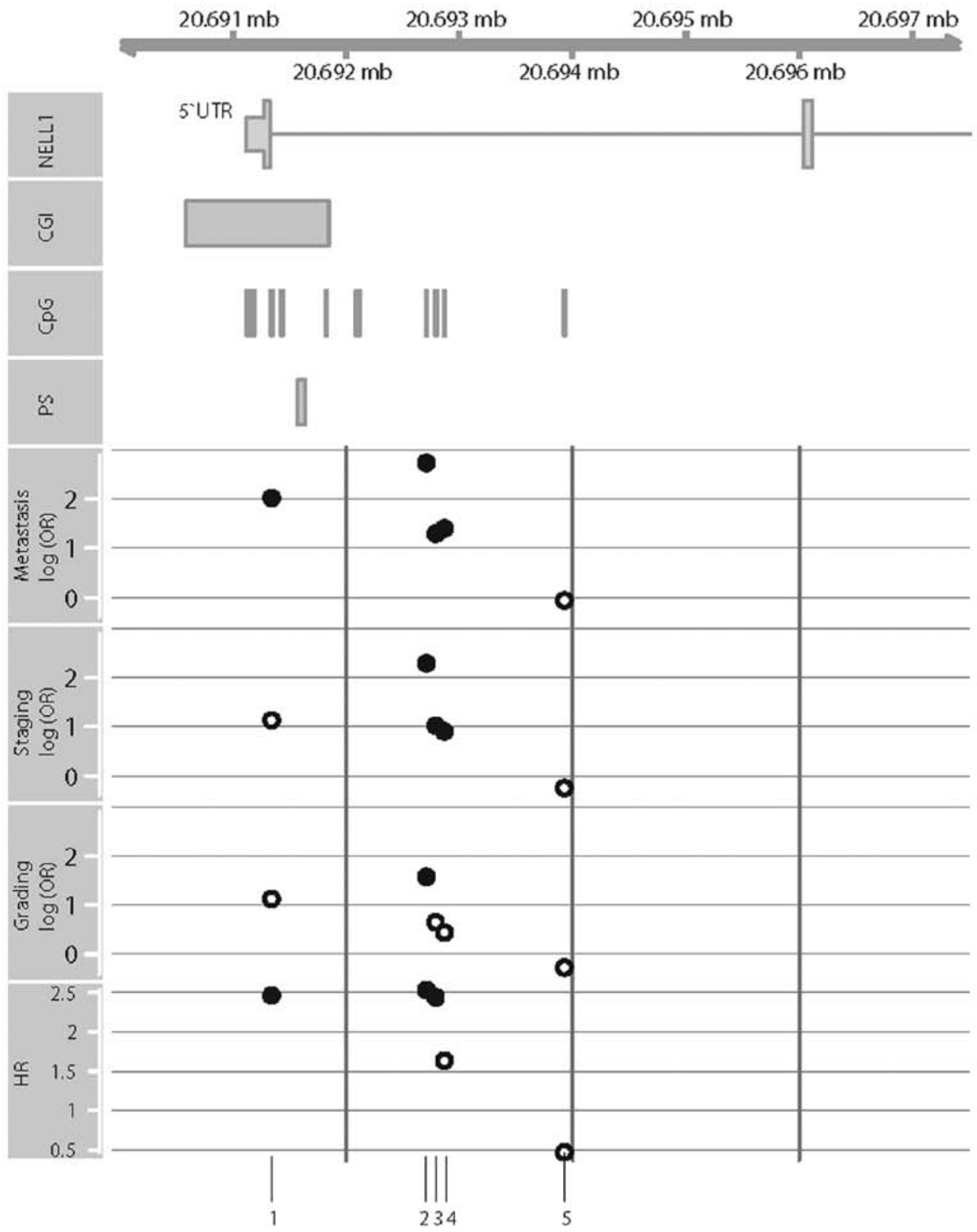

Figure 6. Schematic illustration of the 5'UTR and exon 1 of the NELL1 gene and the allocated CpG island (CGI) and CpG sites (CpG). The blue bar in row four illustrates our pyrosequencing assay (PS), which was used to determine relative methylation. The second part of the Figure shows the statistical associations between NELL1 methylation levels in the KIRC cohort and the status of metastasis, tumor stage, tumor grade, and survival. Circles indicated the investigated $\mathrm{CpG}$ sites in the TCGA cohort: Filled circles show significant associations between hypermethylation and clinicopathology; open circles indicate statistically non-significant associations. We used in silico analyses to investigate these five CpG sites, annotated by the KIRC TCGA consortium (1. cg04261192; 2. $\operatorname{cg} 20764116 ; 3 . \operatorname{cg} 23549358 ; 4$. $\operatorname{cg} 01461552 ; 5$. $\operatorname{cg} 09150140)$, among others (described in the Results section). At a majority of the investigated CpG sites in the TCGA cohort, DNA methylation of NELL1 was highly significantly associated with patient survival (max. HR 2.5; last bar). OR, odds ratio; HR, hazard ratio.

Previous studies indicated that the loss of NELL1 mRNA and concurrent loss of NELL1 protein expression are associated with altered tumor cell behavior. Those findings strongly indicated that epigenetic silencing of the NELL1 gene is functionally relevant (19). Therefore, our finding that high levels of DNA methylation were associated with adverse clinicopathology in patients supported the hypothesis that the loss of NELL1 function promotes the development of aggressive ccRCC.

DNA methylation of NELL1 has also been shown in other human cancers. For example, a high frequency of NELL1 methylation was reported in colon cancer, early-stage esophageal adenocarcinoma (EAC), and gastric cancer (25-27).
Notably, in EAC, NELL1 methylation was significantly associated with a poor prognosis in patients $(25,26)$. No previous study had investigated whether NELL1 methylation might also be correlated with disadvantageous clinical parameters, such as advanced disease, presence of distant metastases, or worse survival in RCC. Therefore, our finding that higher methylation was significantly associated with metastatic and advanced disease in patients with RCC extended the number of human malignancies that showed associations between NELL1 methylation and aggressive tumor biology.

Similarly, our survival analysis indicated that NELL1 methylation could serve as a prognostic indicator of RFS in patients. However, due to our small survival cohort, a 
multivariate analysis was not feasible; due to this limitation, the relevance of this part of the clinical evaluation should be interpreted with caution.

On the other hand, our evaluation of KIRC data confirmed our finding that NELL1 methylation in the CGI and adjacent $\mathrm{CpG}$ sites were associated with the state of distant metastasis, the tumor grade, and the tumor stage. Notably, three of five $\mathrm{CpG}$ sites in this region also showed associations with RFS. However, considering that multivariate significance could not be achieved in the in silico survival analyses, the association between NELL1 methylation and patient survival remains to be confirmed in appropriate study cohorts. Moreover, from a technical point of view, only a small fraction (roughly estimated at about $1 \%$ ) of the totally available $\mathrm{CpG}$ sites could be considered for in silico TCGA methylation analyses. Thus, the combined number of $\mathrm{CpG}$ sites was limited to 25 sites in our experimental and in silico approaches. Hence, most likely, in future, it will be necessary to extend both the study cohorts and the number of target $\mathrm{CpG}$ sites to determine the relevance of NELL1 DNA methylation detection for predicting the clinical course of RCC and for translating these findings into individualized therapy and follow-up regimes.

In conclusion, our analyses suggested that NELL1 DNA methylation is a promising candidate prognostic epigenetic biomarker for more detailed analyses of RCC disease. This finding provides a potential clinical marker to complement previously defined functional data (19). Furthermore, our finding that the prostate and urothelial cancer cell line models demonstrated high relative methylation levels indicated that NELL1 DNA methylation might be also relevant to investigations of other frequent tumor entities. Thus, NELL1 methylation might serve as a potential target structure for investigations with molecular therapeutic objectives.

\section{Acknowledgements}

We thank Christel Reese for the technical assistance.

\section{Funding}

No funding was received for this study.

\section{Availability of data and materials}

All data and materials are available at our local laboratory (Hannover Medical School, 30625 Hannover, Dept. of Urology).

\section{Authors' contributions}

IP wrote the manuscript, prepared the figures, and participated in the study design. ND carried out the methylation analyses and participated in the sequence analyses. JH and IP assembled histopathological, clinicopathological, and survival data. ND and JH isolated and characterized the tissue samples and collected the patients' data. WIA re-evaluated the histopathology, revised the manuscript critically and finally approved the full version. MAK, CAJvK, HT, and AS contributed to the study design, revised the manuscript critically and did the final approval of the version for publication. JS identified the candidate promoter, conceived the study, developed the study design and analytical assays, constructed and ran the clinical database, performed the statistical analyses (together with IP), and participated in the manuscript preparation. All authors read and approved the final manuscript and provided consent for publication.

\section{Ethics approval and consent to participate}

The Ethics Committee of the Eberhard Karls University approved the study (vote no. 128/2003V and 1213-2011) and informed consent was obtained from each patient.

\section{Patient consent for publication}

Not applicable.

\section{Competing interests}

The authors declare no competing interest.

\section{References}

1. Ferlay J, Soerjomataram I, Dikshit R, Eser S, Mathers C, Rebelo M, Parkin DM, Forman D and Bray F: Cancer incidence and mortality worldwide: Sources, methods and major patterns in GLOBOCAN 2012. Int J Cancer 136: E359-E386, 2014.

2. Creighton CJ, Morgan M, Gunaratne PH, Wheeler DA, Gibbs RA, Robertson A, Chu A, Beroukhim R, Cibulskis K, Signoretti S, et al: Comprehensive molecular characterization of clear cell renal cell carcinoma. Nature 499: 43-49, 2013.

3. Cheng L, Zhang S, MacLennan GT, Lopez-Beltran A and Montironi R: Molecular and cytogenetic insights into the pathogenesis, classification, differential diagnosis, and prognosis of renal epithelial neoplasms. Hum Pathol 40: 10-29, 2009.

4. Morris MR, Ricketts C, Gentle D, Abdulrahman M, Clarke N, Brown M, Kishida T, Yao M, Latif F and Maher ER: Identification of candidate tumour suppressor genes frequently methylated in renal cell carcinoma. Oncogene 29: 2104-2117, 2010.

5. Peters I, Rehmet K, Wilke N, Kuczyk MA, Hennenlotter J, Eilers T, Machtens S, Jonas U and Serth J: RASSF1A promoter methylation and expression analysis in normal and neoplastic kidney indicates a role in early tumorigenesis. Mol Cancer 6: 49, 2007.

6. Atschekzei F, Hennenlotter J, Jänisch S, Großhennig A, Tränkenschuh W, Waalkes S, Peters I, Dörk T, Merseburger AS, Stenzl A, et al: SFRPl CpG island methylation locus is associated with renal cell cancer susceptibility and disease recurrence. Epigenetics 7: 447-457, 2012.

7. Onay H, Pehlivan S, Koyuncuoglu M, Kirkali Z and Ozkinay F: Multigene methylation analysis of conventional renal cell carcinoma. Urol Int 83: 107-112, 2009.

8. Ibanez de Caceres I, Dulaimi E, Hoffman AM, Al-Saleem T, Uzzo RG and Cairns P: Identification of novel target genes by an epigenetic reactivation screen of renal cancer. Cancer Res 66: 5021-5028, 2006.

9. Morris MR, Gentle D, Abdulrahman M, Clarke N, Brown M, Kishida T, Yao M, Teh BT, Latif F and Maher ER: Functional epigenomics approach to identify methylated candidate tumour suppressor genes in renal cell carcinoma. Br J Cancer 98: 496-501, 2008.

10. Dubrowinskaja N, Gebauer K, Peters I, Hennenlotter J, Abbas M, Scherer R, Tezval H, Merseburger AS, Stenzl A, Grünwald V, et al: Neurofilament Heavy polypeptide $\mathrm{CpG}$ island methylation associates with prognosis of renal cell carcinoma and prediction of antivascular endothelial growth factor therapy response. Cancer Med 3: 300-309, 2014.

11. Henrique R, Luís AS and Jerónimo C: The epigenetics of renal cell tumors: From biology to biomarkers. Front Genet 3: 94, 2012. 
12. Watanabe TK, Katagiri T, Suzuki M, Shimizu F, Fujiwara T, Kanemoto N, Nakamura Y, Hirai Y, Maekawa $H$ and Takahashi EI: Cloning and characterization of two novel human cDNAs (NELL1 and NELL2) encoding proteins with six EGF-like repeats. Genomics 38: 273-276, 1996.

13. Ting K, Vastardis H, Mulliken JB, Soo C, Tieu A, Do H, Kwong E, Bertolami CN, Kawamoto H, Kuroda S, et al: Human NELL-1 expressed in unilateral coronal synostosis. J Bone Miner Res 14: 80-89, 1999

14. Aghaloo T, Cowan CM, Chou YF, Zhang X, Lee H, Miao S, Hong N, Kuroda S, Wu B, Ting K, et al: Nell-1-induced bone regeneration in calvarial defects. Am J Pathol 169: 903-915, 2006.

15. Maeda K, Matsuhashi S, Tabuchi K, Watanabe T, Katagiri T, Oyasu M, Saito N and Kuroda S: Brain specific human genes, NELL1 and NELL2, are predominantly expressed in neuroblastoma and other embryonal neuroepithelial tumors. Neurol Med Chir 41: 582-589, 2001.

16. Shah US and Getzenberg RH: Fingerprinting the diseased prostate: Associations between BPH and prostate cancer. J Cell Biochem 91: 161-169, 2004.

17. Osman I, Bajorin DF, Sun TT, Zhong H, Douglas D, Scattergood J, Zheng R, Han M, Marshall KW and Liew CC: Novel blood biomarkers of human urinary bladder cancer. Clin Cancer Res 12: 3374-3380, 2006.

18. Slovak ML, Bedell V, Hsu YH, Estrine DB, Nowak NJ, Delioukina ML, Weiss LM, Smith DD and Forman SJ: Molecular karyotypes of Hodgkin and Reed-Sternberg cells at disease onset reveal distinct copy number alterations in chemosensitive versus refractory Hodgkin lymphoma. Clin Cancer Res 17: 3443-3454, 2011.

19. Nakamura R, Oyama T, Tajiri R, Mizokami A, Namiki M, Nakamoto $\mathrm{M}$ and Ooi A: Expression and regulatory effects on cancer cell behavior of NELL1 and NELL2 in human renal cell carcinoma. Cancer Sci 106: 656-664, 2015.
20. Gebauer K, Peters I, Dubrowinskaja N, Hennenlotter J, Abbas M, Scherer R, Tezval H, Merseburger AS, Stenzl A, Kuczyk MA, et al: Hsa-mir-124-3 CpG island methylation is associated with advanced tumours and disease recurrence of patients with clear cell renal cell carcinoma. Br J Cancer 108: 131-138, 2013.

21. Sobin LH and Compton CC: TNM seventh edition: What's new, what's changed: Communication from the international union against cancer and the American Joint Committee on cancer. Cancer 116: 5336-5339, 2010.

22. Peters I, Eggers H, Atschekzei F, Hennenlotter J, Waalkes S, Tränkenschuh W, Grosshennig A, Merseburger AS, Stenzl A, Kuczyk MA, et al: GATA5 CpG island methylation in renal cell cancer: A potential biomarker for metastasis and disease progression. BJU Int 110: E144-E152, 2012.

23. Colella S, Shen L, Baggerly KA, Issa JP and Krahe R: Sensitive and quantitative universal Pyrosequencing methylation analysis of CpG sites. Biotechniques 35: 146-150, 2003.

24. Team RDC: R Development Core Team. 2011. R: A language and environment for statistical computing. $\mathrm{R}$ Foundation for Statistical Computing, Vienna, Austria. Available at http:// www.R-project.org/.

25. Mori Y, Cai K, Cheng Y, Wang S, Paun B, Hamilton JP, Jin Z, Sato F, Berki AT, Kan T, et al: A genome-wide search identifies epigenetic silencing of somatostatin, tachykinin-1, and 5 other genes in colon cancer. Gastroenterology 131: 797-808, 2006.

26. Jin Z, Mori Y, Yang J, Sato F, Ito T, Cheng Y, Paun B, Hamilton JP, Kan T, Olaru A, et al: Hypermethylation of the nel-like 1 gene is a common and early event and is associated with poor prognosis in early-stage esophageal adenocarcinoma. Oncogene 26 : 6332-6340, 2007.

27. Gao C, Zhang Q, Kong D, Wu D, Su C, Tong J, Chen F and Zhang Q: MALDI-TOF Mass Array analysis of Nell-1 promoter methylation patterns in human gastric cancer. Biomed Res Int 2015: 136941, 2015. 